\title{
Aberraciones cromosómicas en trabajadoras expuestas a plaguicidas
}

\author{
Patricia Cuenca ${ }^{1,2 *}$ \& Vanessa Ramírez ${ }^{1,3}$ \\ Instituto de Investigaciones en Salud (INISA), Universidad de Costa Rica, 2060, San José, Costa Rica. \\ Escuela de Biología, Universidad de Costa Rica, 2060, San José, Costa Rica. \\ Escuela de Nutrición, Universidad de Costa Rica, 2060, San José, Costa Rica. \\ * Correspondencia: Instituto de Investigaciones en Salud (INISA), Universidad de Costa Rica, San José, Costa Rica, \\ Tel (506) 224-3668, Fax (506) 207-5130; pcuenca@cariari.ucr.ac.cr
}

Recibido 17-VI-2004. Corregido 09-VIII-2004. Aceptado 23-VIII-2004.

\begin{abstract}
Chromosomic aberrations in female workers exposed to pesticides. The purpose of this work was to determine if the occupational exposure to those pesticides used at banana plantations' packaging plants produces genetic damage to somatic cells of female workers. Chromosomal aberrations were scored in lymphocytes of 20 women, 10 female exposed workers and 10 female controls. Workers were recruited from independent farms from two locations in Costa Rica, during January through June in 1996 and 1997. These females had a minimum of three months of work, had never received chemotherapy or radiotherapy and did some of these labors: sealing, spraying or weighting of bananas. Control unexposed females lived in the same area, were of similar age and neither them nor their husbands/mates had ever worked in pesticide related labors. For each female, 100 mitotic figures were scored. The kind of aberrations detected were acentric fragments, dicentric chromosomes, rings, gaps and breaks. Among workers, $16 \%$ of cells $(\mathrm{n}=1000)$ had one or more abnormalities, whereas control unexposed females had $6 \%$ of cells $(n=1000)$ with comparable anomalies $(\mathrm{p}<0.05)$. In conclusion, the pesticide exposure is a risk factor for chromosome aberrations in female somatic cells. Rev. Biol. Trop. 52(3): 623-628. Epub 2004 Dic 15.
\end{abstract}

Key Words: pesticides, chromosomal aberrations, Costa Rica, banana parking.

Palabras clave: pesticidas, aberraciones cromosómicas, Costa Rica, empacado de banano.

Los trabajadores de las fincas y empacadoras de banano en Costa Rica se encuentran expuestos a plaguicidas en mayor grado que los agricultores de otros cultivos y los agricultores de los países templados; su exposición ocurre a través de todo el año (Wesseling et al. 1996, Ramírez y Cuenca 2001, 2002, García 2003, Castro et al 2004). Entre los problemas de salud a largo plazo que puede causar dicha exposición, se encuentra el aumento del riesgo para el desarrollo de tumores en los mismos trabajadores o en sus hijos (Fear 1998); y problemas reproductivos, tales como abortos repetidos (Savitz et al. 1994). En los trabajadores costarricenses, existen los antecedentes de esterilidad por exposición al DBCP (Ramírez y
Ramírez 1980) y de una mayor tasa de incidencia estandarizada de cáncer de pene y melanoma de extremidades inferiores en varones trabajadores bananeros, así como de leucemia en las trabajadoras (Wesseling et al. 1996).

La Agencia Internacional para la Investigación del Cáncer (IARC por sus siglas en Inglés) ha identificado una serie de procesos industriales y ocupaciones asociados a un riesgo aumentado de desarrrollar algún tumor o tumores (Pearce et al. 1994). La exposición ocupacional a plaguicidas no arsenicados está clasificada por la IARC como probablemente carcinogénica (Grupo $2 \mathrm{~A}$ ), esto significa que este tipo de exposición es carcinogénica para animales y que no existe la certeza suficiente 
para clasificarla como carcinogénica para humanos (Anónimo 1994), lo cual muestra la necesidad de documentar sus efectos con más investigaciones.

Las aberraciones cromosómicas son encontradas frecuentemente en las células cancerosas, en los productos de abortos espontáneos y en muchos individuos con desórdenes del desarrrollo. La fuerte asociación que existe entre aberraciones cromosómicas y la formación de tumores hace que su análisis en poblaciones de alto riesgo sea considerado un método apropiado para estudiar clastogenicidad y se consideran un buen predictor de carcinogenicidad (Nordenson et al. 1984, Sorsa et al. 1992, Hagmar et al. 1994, 1998, Au et al. 1999, Preston 1999, Bonassi y Au 2002). Tanto las sustancias químicas que reaccionan directamente con el ADN, como algunas de las consideradas mutágenos indirectos, llamados equivocadamente no genotóxicos, pueden producir aberraciones cromosómicas (Preston 1999). Poblaciones expuestas a sustancias químicas han sido monitoreadas en diversas y numerosas investigaciones. Los hallazgos positivos para aberraciones cromosómicas indican exposición excesiva al agente y sugieren peligro de daño a la salud de estas personas. Este valor predictivo no se ha encontrado para intercambio de cromátidas hermanas ni para los micronúcleos (Bonassi y Au 2002). La IARC usó datos derivados del monitoreo de poblaciones humanas en la clasificación del óxido de etileno como un carcinógeno humano. Se consideró que los datos citogenéticos brindaban una base para esa conclusión (Anónimo 1994).

Un aumento en la frecuencia de aberraciones cromosómicas en trabajadores expuestos a mezclas de plaguicidas ha sido informado por varios estudios; sin embargo, los hallazgos no son consistentes en todos ellos debido a las diferentes variables que influyen en los resultados, tales como el tipo de plaguicida y la duración y la intensidad de la exposición (Au et al. 1999).

En síntesis, la presencia de las aberraciones cromosómicas en los linfocitos es el mejor biomarcador de predicción para potenciales problemas de salud como cáncer y abortos en poblaciones expuestas a mutágenos y cancerígenos. La experiencia del uso de este marcador indica que es uno de los ensayos más sensibles y relevantes para la identificación de mutágenos, carcinógenos y teratógenos en grupos humanos expuestos ( $\mathrm{Au} \mathrm{1991,} \mathrm{Au} \mathrm{y}$ Sram 1996, Preston 1999, Bonassi y Au 2002, Cuenca y Ramírez 2004).

El presente trabajo se llevó a cabo con el objetivo de conocer si la exposición ocupacional a los plaguicidas utilizados en las plantas empacadoras de banano, provoca daño en los cromosomas de las células somáticas de las trabajadoras expuestas.

\section{MATERIALES Y MÉTODOS}

Se invitaron a participar en la investigación a las trabajadoras que estaban laborando en plantas empacadoras de banano independientes, localizadas en los cantones de Guápiles y Siquirres, Costa Rica. Se incluyeron aquellas mujeres con un mínimo de tres meses de antigüedad en la finca, que en el momento de ser reclutadas trabajaban sellando, pesando o atomizando la fruta; y que nunca hubieran recibido radioterapia o tratamiento con citotóxicos. La participación voluntaria quedó plasmada en un formulario de consentimiento informado. Se les hizo una entrevista con un cuestionario diseñado y utilizado internacionalmente para este tipo de estudios (Carrano y Natarajan 1988), adaptado y validado previamente mediante su aplicación a un grupo de mujeres de la misma región que no participaron en este estudio. Se les preguntó sobre edad, historia laboral, exposición a plaguicidas y otras sustancias, antecedentes de salud, ingesta de medicamentos, exposición a rayos $\mathrm{X}$, número de hijos, número de abortos espontáneos, hábitos de fumado e ingesta de bebidas alcohólicas. Se estudió también un grupo de mujeres de la comunidad, con las mismas edades, que no hubieran trabajado nunca en agricultura y cuyos esposos o compañeros no trabajaran con plaguicidas. Se constituyó un 
grupo de diez trabajadoras expuestas y diez no expuestas. En este tipo de estudios se considera más importante homogeneizar el grupo de casos con el grupo control con respecto a las variables confundentes, que utilizar una muestra grande de casos (Preston 1999).

Se colectó sangre en heparina de sodio y antes de cumplirse 24 horas de tomada la muestra se cultivaron linfocitos en medio RPMI suplementado con suero bovino fetal al $10 \%$. Las preparaciones para observación microscópica fueron codificadas por un colaborador que no participó en el análisis. De cada persona se analizaron 100 metafases, para las cuales se registraron las fracturas, lagunas, fragmentos acéntricos, cromosomas dicéntricos y anillos.

Se aplicó un análisis de varianza univariado para comparar el grupo de expuestas con el grupo control con respecto a las variables confundentes investigadas por medio de la entrevista. Para comparar las anormalidades cromosómicas se realizó la prueba T de Student para grupos independientes, $\mathrm{p} \leq 0.05$ (Hernández y Bolaños 1993).

\section{RESULTADOS}

Las trabajadoras del empaque de banano en Costa Rica están expuestas directamente a imazalil y tiabendazol (fungicidas que se aplican a la fruta); además del clorpirifós (insecticida impregnado en las bolsas que protegen los racimos en el campo). También se encontraban indirectamente expuestas al resto de los plaguicidas que se estaban utilizando en esas fincas en el momento del muestreo, según la información extraída de los expedientes del Departamento de sustancias tóxicas y medicina del trabajo del Ministerio de Salud: cadusafós, carbofurán, terbufós, oxamil, glisofato, paraquat, ametrina, diuron, Bacillus thuringensis, diazinon, Dipterex, Neguvon, formalina, bromuro de metilo, Beloran, Banodine, mancozeb, benomil, tridemorph, maneb, clorotalonil, propiconazol, Calcium, Dithar, Tilt, Calixin, Vondozeb.
El análisis de varianza entre los dos grupos estudiados mostró diferencia únicamente para la variable exposición a plaguicidas. Para las variables con un potencial efecto confundente sobre la frecuencia de aberraciones cromosómicas no hubo ninguna diferencia. Esto confirma que tanto las mujeres expuestas como las no expuestas fueron similares en aspectos como edad, fumado, ingesta de alcohol, café, té, medicamentos, uso de anticonceptivos orales, antecedentes de infecciones bacterianas y virales en los meses previos al estudio, cantidad de radiografías, enfermedad cardiovascular, dermatitis y otras alergias, cirugías en el último año, antecedentes de abortos espontáneos, mortinatos e hijos con malformaciones congénitas. El rango de edad del grupo en estudio es de 20 a 46 años; y el ámbito de exposición de las trabajadoras es de 36 a 240 meses.

En el cuadro 1 se muestra la cantidad de anormalidades cromosómicas encontradas para todas las parejas de expuestas-no expuestas, así como también la significancia obtenida con la prueba de t-Student. Se aprecia que las trabajadoras expuestas presentaron una mayor frecuencia de fracturas cromosómicas, total de anormalidades y número de células anormales con $\mathrm{p} \leq 0.05$.

\section{DISCUSIÓN}

Las investigaciones sobre efecto genotóxico en las poblaciones humanas, basadas en parámetros genéticos deben controlar algunas variables confundentes, conocidas por aumentar la frecuencia de daño cromosómico y de la molécula de ADN. Entre estas variables se encuentran la edad, la exposición a rayos $\mathrm{X}$, el fumado, el consumo de café y los tratamientos anticáncer, ya sea quimioterapia o radioterapia (Carrano 1988, Ashby et al. 1993, Au et al. 1991, Preston 1999 ). No es posible tomar valores de referencia de la población general, ya que las variables a evaluar están sujetas a factores como estilo de vida y a las condiciones de trabajo propias de cada laboratorio. Por esta razón, se debe trabajar con un grupo testigo lo 


\section{CUADRO 1}

Aberraciones cromosómicas en trabajadoras expuestas a plaguicidas en las empacadoras de banano y controles de mujeres no expuestas, apareadas por edad, con un promedio de 172 meses de trabajo

TABLE 1

Chromosomal aberrations in female workers exposed to pesticidas at the banana packaging sites and in female unexposed controls, paired by age, with a mean of 172 months of work

\begin{tabular}{lccccccc} 
& \multicolumn{2}{c}{ Total } & \multicolumn{2}{c}{ Promedio } & \multicolumn{2}{c}{ Desv. Estándar } & Significancia \\
& E & NE & E & NE & E & NE & \\
Comosomas dicéntricos & 2 & 2 & 0.2 & 0.2 & 0.63 & 0.63 & N.S. \\
Anillos cromosómicos & 1 & 0 & 0.1 & - & 0.32 & - & N.S. \\
Fragmentos acéntricos & 3 & 14 & 0.3 & 1.4 & 0.94 & 1.58 & N.S. \\
Fracturas cromosómicas & 47 & 16 & 4.7 & 1.6 & 3.77 & 1.65 & p $<0.05$ \\
Lagunas cromosómicas & 113 & 39 & 11.3 & 3.9 & 11.63 & 4.30 & N.S. \\
Total de anormalidades & 166 & 65 & 16.6 & 6.5 & 13.80 & 5.85 & p $=0.05$ \\
Total de células anormales & 159 & 58 & 15.9 & 5.8 & 13.40 & 5.82 & p $<0.05$
\end{tabular}

E: expuestas; NE.: no expuestas; N.S. diferencia no significativa; $\mathrm{p} \leq 0,05$ diferencia significativa.

más similar posible al grupo en estudio, así como, manejar las muestras de los individuos en estudio bajo condiciones estandarizadas.

Las trabajadoras mostraron mayor frecuencia en fracturas cromosómicas, cantidad total de células anormales y total de anormalidades cromosómicas. Resultados similares han sido previamente encontrados por otros autores. En 1973 se informó por primera vez que los trabajadores que aplican plaguicidas presentaban daño cromosómico durante la estación de mayor aplicación, posteriormente también se encontró aumentada la frecuencia de fracturas cromosómicas en trabajadores intoxicados con organofosforados. Trabajadores de las fábricas de DDT en Brasil y floricultores de Argentina también mostraron mayor frecuencia de aberraciones cromosómicas (Dulout et al. 1985). El único estudio de este tipo realizado en trabajadores costarricenses fue llevado a cabo por investigadores de la Universidad de Texas. Incluyeron veinte hombres afectados de problemas reproductivos causados por la exposición laboral al DBCP, no encontraron diferencia significativa entre casos y controles, $1.6 \%$ de células anormales contra el $1.26 \%$ del grupo control (Au et al. 1999). La exposición a este plaguicida había ocurrido en los años sesentas y setentas, por lo que la mayoría de los sujetos estudiados ya estaban retirados de las labores agrícolas cuando se analizaron sus cromosomas. La disminución de la exposición a la sustancia genotóxica causa con el tiempo, una disminución de la frecuencia de anormalidades cromosómicas, lo cual ha sido comprobado por otros autores (Preston 1999).

La ejecución de estudios prospectivos de esta naturaleza es muy difícil de hacer; por lo que existen muy pocas publicaciones con datos que documenten efecto genotóxico en varios momentos de la vida de los mismos individuos, sin embargo los estudios realizados en el norte de Europa y en Italia han demostrado que las aberraciones cromosómicas constituyen el mejor biomarcador de efecto temprano para predecir riesgo de desarrollar cáncer (Preston 1999, Bonassi y Au 2002). Debemos recordar que el estudio sobre cáncer desarrollado en Costa Rica por Wesseling et al. (1996) en trabajadores bananeros, encontró riesgo aumentado para leucemias en las mujeres.

Desde el punto de vista del efecto genotóxico de los plaguicidas, ésta es la primera experiencia realizada en un laboratorio del país. Consideramos de suma importancia continuar con este tipo de estudios ya que en Costa Rica se están utilizando una larga lista deplaguicidas 
que han sido prohibidos por su toxicidad, no solamente en países desarrollados, sino también en países del Istmo Centroamericano, como Belice y Panamá (Vaquerano 2001, García 2003), por lo que es necesario documentar científicamente los efectos de las exposiciones laborales a estas sustancias, con el fin de aportar elementos objetivos que contribuyan a la toma de mejores decisiones. Estudios similares a este se deben llevar a cabo con muestras de mayor tamaño que permitan realizar análisis multivariado de los datos.

Los resultados de este trabajo reafirman que la exposición ocupacional a los plaguicidas causa daño al material genético de las personas expuestas, por lo que constituyen un riesgo para la salud. El estado, como responsable de velar por la salud pública, debería ser más estricto con los controles sobre las medidas de seguridad que establece la ley para proteger a este sector de trabajadores. Se reafirma la importancia de legislar en Costa Rica para prohibir el uso de aquellos plaguicidas que ya lo están en otras partes del mundo.

\section{AGRADECIMIENTOS}

Al personal del INISA, especialmente a Federico Hernández por la asistencia técnica. Este trabajo se realizó con el aporte financiero de la Vicerrectoría de Investigación de la Universidad de Costa Rica N ${ }^{\circ}$ 742-95-277, PLAGSALUD/OPS, CONICIT y el Servivio Alemán de Intercambio Académico (DAAD).

\section{RESUMEN}

Con el objetivo de conocer si la exposición ocupacional a los plaguicidas utilizados en las plantas empacadoras de banano provoca daño genético en las células somáticas de las trabajadoras, se estudió la frecuencia de aberraciones cromosómicas en linfocitos de un grupo de diez trabajadoras expuestas y diez mujeres no expuestas. Las trabajadoras se captaron en fincas independientes de los cantones de Guácimo y Pococí, Costa Rica, entre enero y junio de los años 1996 y 1997. El grupo de expuestas tenían un mínimo de tres meses de trabajo, no habían recibido quimioterapia o radioterapia, y realizaban alguna de las siguientes labores: sellar, atomizar y/o pesar la fruta. El grupo de no expuestas estuvo formado por mujeres residentes de la misma zona, con edades similares; tanto ellas como sus esposos o compañeros no trabajaban en labores relacionadas con plaguicidas. Se analizaron 100 figuras mitóticas por persona y se registraron las aberraciones siguientes: fragmentos acéntricos, cromosomas dicéntricos, anillos, lagunas y fracturas cromosómicas. El $16 \%$ de las células $(n=1000)$ pertenecientes a las trabajadoras tenían una o más anormalidades contra el $6 \%$ $(n=1000)$ en el grupo de mujeres no expuestas $(p \leq 0.05)$.

\section{REFERENCIAS}

Anónimo. 1994. Occupational cancer in developing countries. IARC Scientific. Publications $N^{\circ} 129$. Lyon. 191 p.

Ashby, J., W. Angbar, W. Au, A. Massoud \& J. Gentile. 1993. Genetic Toxicology in Developing Countries: Comments and recommendations. Environ. Health Perspect. 101: 335-338.

Au, W. 1991. Monitoring human populations for effects of radiation and chemical exposures using cytogenetics techniques. Ocupational Med. 6: 597-611.

Au, W., D.M. Walker, J.B.Ward, E. Whorton, M.Legator \& V. Singh. 1991. Factors contributing to chromosome damage in lymphocytes of cigarette smokers. Mut. Res. 260: 137-144.

Au, W. \& R. Sram. 1996. Second International Conference on Environmental Mutagens in Human Populations. Environ Health Perspect 104 (Suppl 3): 421-422.

Au, W., C.H. Sierra-Torres, N. Cajas-Salazar, B.K. Shipp \& M. S. Legator. 1999. Cytogenetic effects from exposure to mixed pesticides and the infkuence from genetic susceptibility. Environ. Health Perspect. 107: 501-505.

Bonassi, S. \& W.W. Au. 2002. Biomarkers in molecular epidemiology studies for health risk prediction. $\mathrm{Mu}-$ tat. Res. 511: 73-86.

Carrano, A.V. \& A.T. Natarajan. 1988. Consideration for population monitoring using cytogenetic techniques. International Commision for Protection against environmental mutagens and carcinogens. Publication No 14. Mut. Res. 204: 378-406.

Castro, R., V. Ramírez \& P. Cuenca. 2004. Análisis de micronúcleos y otras anormalidades nucleares en el epitelio oral de mujeres expuestas ocupacionalmente a plaguicidas. Rev. Biol. Trop. 52: 207-217. 
Cuenca, P. \& V. Ramírez. 2004. Mutagénesis ambiental y el uso de biomarcadores para predecir y prevenir el riesgo de cáncer. 52: 181-186.

Dulout, F.N., M.C. Pastori, A.O. Olivero, M. González Cid, D. Loría, E. Matos, N. Sobel, E.C. de Buján \& N. Albiano. 1985. Sister-chromatid exchanges and chromosomal aberrations in a population exposed to pesticides. Mut. Res. 143: 237-244.

Fear, N.T. 1998. Childhood cancer and paternal employment in agriculture: the role of pesticides. Br. J. Cancer 77: 825-829.

García, J. 2003. Plaguicidas: Problemática y opciones de solución, con énfasis en Costa Rica. Foro Latinoamericano. Acta Académica 33: 33-52.

Hagmar, L., A. Brogger, I.L.Hansteen, S. Heim, B. Högstedt, L. Knudsen, B.Lambert, K. Linnainmaa, F. Mitelman, I. Nordenson, Ch. Reuterwall, S. Salomaa, S. Skerfving \& M. Sorsa. 1994. Cancer risk in human predicted by increased levels of chromosomal aberrations in lymphocytes: Nordic Study Group on the Health Risk of Chromosome Damage. Cancer Res. 54: 2919-2922.

Hagmar, L., S. Bonassi, U. Stromberg, A. Brogger, L. Knudsen, H. Norppa, C. Reuterwall \& The European Study Group on Cytogenetic Biomarkers and Health. 1998. Chromosomal aberrations in lymphocytes predict human canmcer: A report from the European Study Group on Cytogenetics Biomarkers and Health (ESCH). Cancer Res. 58: 4117-4121.

Hernández,O. \& R.L. Bolaños. 1993. SPSS/PC + básico. Editorial de la Universidad de Costa Rica. San José, $249 \mathrm{p}$.

Nordenson, I., L.Beckman, S. Liden \& N. Stjernberg. 1984. Chromosomal Aberration and Cancer Risk. Hum. Hered. 34: 76-81.
Pearce, N., E. Matos, H.Vainio, P. Boffetta, M. Kogevinas (eds.). 1994. Occupation cancer in developing countries. IARC Scientific Publication. Lyon, 141 p.

Preston, R.J. 1999. Chromosomal changes, pp. 395-408. In D.B. McGregor, J.M. Rice \& S. Venitt. (eds.). The use of short- and medium-term test for carcinogens and data on genetic effects in carcinogenic Hazard evaluation. IARC Scientific Publications $\mathrm{N}^{\circ}$ 146. Lyon.

Ramírez, A.L. \& C.M. Ramírez. 1980. Esterilidad masculina causada por la exposición laboral al nematicida 1,2-Dibromo-3-Cloropropano. Act. Méd. Cost. 23: 219-222.

Ramírez, V. \& P. Cuenca. 2001. Micronuclei frecuency in lymphocytes of individuals occupationally exposed to pesticides. Rev. Biol. Trop 49: 1-8.

Ramírez V. \& P. Cuenca. 2002. Daño del ADN en trabajadoras bananeras expuestas a plaguicidas en Limón, Costa Rica. Rev. Biol. Trop. 50: 507-518.

Savitz, D.A., N.L. Sonnenfeld \& A.F. Olshan. 1994. Review of Epidemiologic Studies of Paternal Occupational Exposure and Spontaneous Abortion. Am. J. Industrial Med. 25: 361-383.

Sorsa, M., J. Wibourn \& H. Vainio. 1992. Human Cytogenetic damage as a predict of cancer risk, pp 543-544. In Vainio, H., P.N. Magee, D.B. McGregor \& A.J. Mc Michael (eds.). Mechanism of carcinogénesis in risk identification, IARC Scientific Publications, Lyon.

Vaquerano, B. 2001. Establecimiento de controles normativos (restricciones y prohibiciones) a los plaguicidas sintéticos. Memoria del foro sectorial: "El uso de plaguicidas y su relación con el desarrollo en Costa Rica”. Proyecto PLAGSALUD, OPS / OMS, RelUITA, Foro Emaús. San José, 66 p.

Wesseling, C., A. Ahlbom, D. Antich, A.C. Rodriguez \& R. Castro. 1996. Cancer in banana plantation workers in Costa Rica. Int. J. Epidemiol. 24: 1125-1131. 